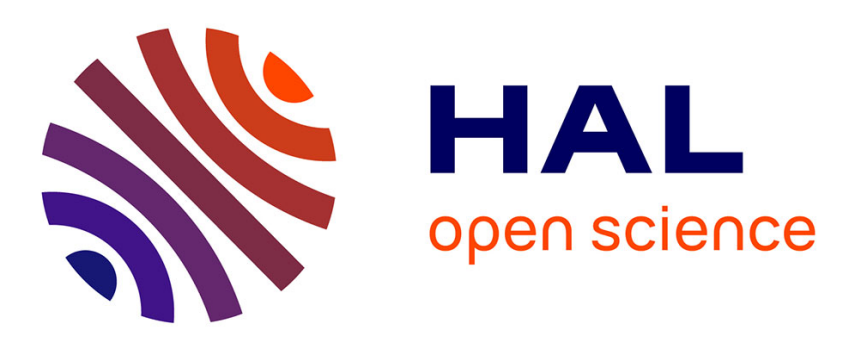

\title{
Tiled top-down pyramids and segmentation of large histological images
}

\author{
Romain Goffe, Luc Brun, Guillaume Damiand
}

\section{To cite this version:}

Romain Goffe, Luc Brun, Guillaume Damiand. Tiled top-down pyramids and segmentation of large histological images. In 8th IAPR - TC-15 Workshop on Graph-based Representations in Pattern Recognition (GBR'11), May 2011, Munster, Germany. pp.255-264. hal-00596703

\section{HAL Id: hal-00596703 https://hal.science/hal-00596703}

Submitted on 29 May 2011

HAL is a multi-disciplinary open access archive for the deposit and dissemination of scientific research documents, whether they are published or not. The documents may come from teaching and research institutions in France or abroad, or from public or private research centers.
L'archive ouverte pluridisciplinaire HAL, est destinée au dépôt et à la diffusion de documents scientifiques de niveau recherche, publiés ou non, émanant des établissements d'enseignement et de recherche français ou étrangers, des laboratoires publics ou privés. 


\title{
Tiled top-down pyramids and segmentation of large histological images
}

\author{
Romain Goffe ${ }^{1}$, Luc Brun ${ }^{1}$, and Guillaume Damiand ${ }^{2}$ \\ 1 GREYC, ENSICAEN, CNRS, UMR6072, F-14050, Caen, France \\ \{romain.goffe, luc.brun\}@greyc.ensicaen.fr \\ 2 LIRIS, Université de Lyon, CNRS, UMR5205, F-69622, Villeurbanne, France \\ guillaume.damiand@liris.cnrs.fr
}

\begin{abstract}
Recent microscopic imaging systems such as whole slide scanners provide very large (up to 18GB) high resolution images. Such amounts of memory raise major issues that prevent usual image representation models from being used. Moreover, using such high resolution images, global image features, such as tissues, do not clearly appear at full resolution. Such images contain thus different hierarchical information at different resolutions. This paper presents the model of tiled top-down pyramids which provides a framework to handle such images. This model encodes a hierarchy of partitions of large images defined at different resolutions. We also propose a generic construction scheme of such pyramids whose validity is evaluated on an histological image application.
\end{abstract}

Key words: Irregular pyramid; Topological model; Combinatorial map;

\section{Introduction}

The increasing amount of high resolution images raises new and major issues in the field of image analysis and processing. For instance, microscopic scanners have recently been improved to the point where whole slide imaging techniques may offer a $\times 40000$ magnification. However, the segmentation of such images requires to handle a hierarchy of large partitions defined on up to 18GB data volumes. A suitable model to encode segmentation of such images should be compatible with memory constraints induced by this large amount of data and should allow to design segmentation algorithms based on the same top-down analysis scheme than pathologists.

In this regard, we identified two key steps during pathologists' manual analysis of pyramids of histological images. First, an identification of histological components for each resolution is performed and regions of interest are determined according to topological or geometric features. Second, these regions of interest are used within a hierarchical scheme from the lowest to the highest resolution (top-down), each region being analyzed in the context defined by its ancestors.

As a consequence, a model with geometrical, topological and hierarchical features is required to provide a segmentation of large histological images. Usual non hierarchical models are devoted to few partitions' properties and do not provide a full 
encoding of geometrical and topological properties of a partition. For example, RAG data structures do not provide an efficient access to the geometry of regions' borders. This drawback is addressed by more sophisticated models such as topological maps [2,3] which encode both geometric and topological properties of a partition.

Hierarchical models such as quadtrees or regular pyramids are commonly used for multi-resolution images representation and segmentation. Yet, both frameworks entail several drawbacks [1]. For example, they may not preserve adjacency of connected regions through different levels of a pyramid. Those drawbacks lead to the design of irregular pyramids [2,3] in order to take advantage of the efficiency of graphs and topological maps for geometric and topological operations while keeping the advantages of their regular ancestors.

We have previously introduced the tiled maps model [4], defined as a topological map decomposed into topological tiles to encode partitions of large images. In [5], we have proposed an efficient construction scheme of tiled top-down pyramids. Using such a construction scheme, each partition defined at a given resolution is initialised by the projection of the partition defined at the previous (lower) resolution and then further refined by split and merge operations. In this paper, we focus on three main points. We first provide an improved formalism to define our model of tiled top-down pyramid (Section 2). We propose in Section 3 a new projection step of each partition which takes into account the additional information provided by the current resolution. We finally show in Section 4 the efficiency of our model with the segmentation of an histological image.

\section{Top-down framework}

\section{$2.1 \quad$ Topological maps}

A topological map model $[2,3]$ encodes both topological and geometrical properties of a partition. It combines three distinct models: a 2-map that encodes topological relationships, a matrix of interpixel elements [8] that encodes the geometry of the partition and a tree of regions for inside relationships (Figure 1).

A combinatorial map is defined from a set of abstract basic elements called darts. Two operators denoted by $\beta_{i}, i \in\{1,2\}$ that apply on darts allow to represent adjacency relationships between cells (Definition 1 ).

Definition 1 (Combinatorial map). In two dimensions, a combinatorial map $M$ is a triplet $M=\left(\mathcal{D}, \beta_{1}, \beta_{2}\right)$ where:

1. $\mathcal{D}$ is a finite set of darts;

2. $\beta_{1}$ is a permutation ${ }^{1}$ on $\mathcal{D}$;

3. $\beta_{2}$ is an involution ${ }^{2}$ on $\mathcal{D}$.

\footnotetext{
${ }^{1}$ A permutation is a one to one mapping from $S$ onto $S$.

${ }^{2}$ An involution $f$ is a one to one mapping from $S$ onto $S$ such that $f=f^{-1}$.
} 


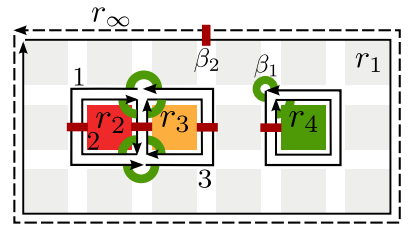

(a)

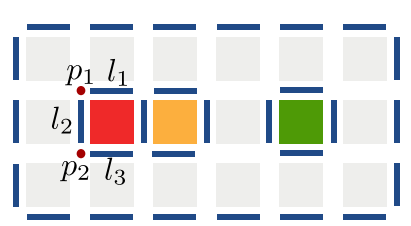

(b)

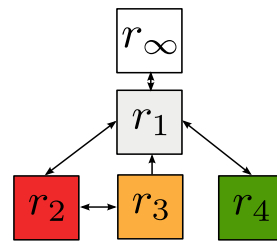

(c)

Figure 1: Topological maps: a combination of three models for the representation of a partition. (a) Combinatorial map for adjacency relationships. Dotted darts belong to the infinite region. (b) Interpixel representation for geometrical borders: bounding pointels and linels are drawn as circles and bold segments. (c) Tree of regions for inside/contains relationships.

Intuitively, a combinatorial map may be considered as a planar graph whose edges are decomposed into half edges called darts. Each dart is incident to a single vertex and belongs to a single edge and a single face. Sets of darts defining high level entities such as vertices, edges and faces are retrieved using cycles $^{3}$ of $\beta_{i}$ permutations. In practice, the $\beta_{1}$ permutation connects a dart of a face to the next dart encountered when turning clockwise around it. The involution $\beta_{2}$ separates two adjacent faces and maps a dart of an edge to the only dart with an opposite orientation which belongs to the same edge. For instance, in Figure $1(\mathrm{a}), \beta_{1}(1)=3$ and $\beta_{2}(1)=2$. Vertices, edge and faces of a combinatorial map are respectively encoded by the cycles of the permutations $\beta_{1} \circ \beta_{2}, \beta_{2}$ and $\beta_{1}$. All faces of a combinatorial map but one encode finite faces (faces corresponding to regions with a finite area). The face which does not satisfy this property encodes the background of the partition encoded by the combinatorial map and is called an infinite face. A combinatorial map is called minimal in number of cells if it does not contain any vertex with a degree lower or equal to 2 and removing any dart would change the topology.

Matrix of interpixel elements Using interpixel elements [8], the geometry of an an $n \times m$ image partition is encoded by an $(n+1) \times(m+1)$ array of marks. Each entry of this array encodes the existence of a given linel (or crack) element within the set of linels encoding image's boundaries and called bounding linels (Figure 1(b)). Note that both pointels incident to a same bounding linel are considered as bounding elements. We call embedding the association of bounding cells with a topological element (vertex, edge or face). In Figure 1(b), embedding of dart 1 is defined as the sequence of bounding elements $\left(l_{1}, p_{1}, l_{2}, p_{2}, l_{3}\right)$. A region corresponds to the embedding of a face and is a set of adjacent pixels.

Tree of regions The tree of regions describes inside/contains relationships: a region is the father of the regions it contains. In Figure 1(c), $r_{1}$ contains $\left\{r_{2}, r_{3}, r_{4}\right\}$.

\footnotetext{
${ }^{3} \mathrm{~A}$ cycle of a permutation is defined as its restriction to the set of images of an element.
} 


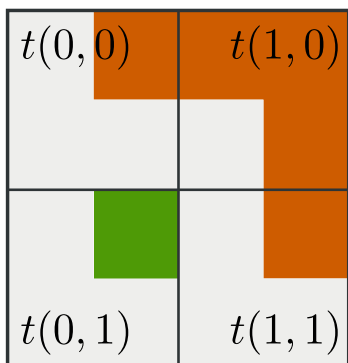

(a)

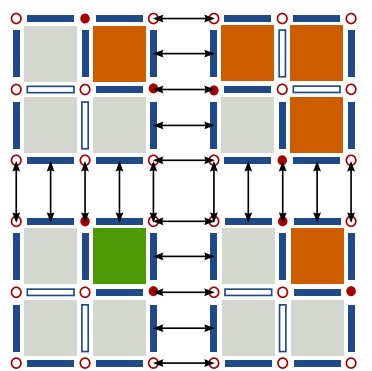

(b)

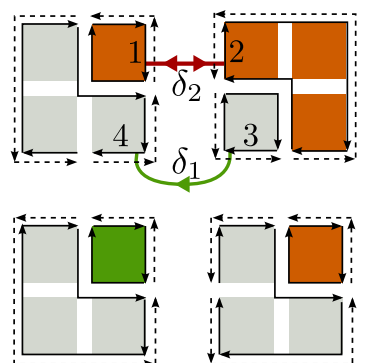

(c)

Figure 2: Tiled maps: an extension to the topological map model designed for large partitions. (a) Original image decomposed into four tiles. (b) Interpixel representation: arrows map equivalent bounding cells. (c) Tiled map: each tile is encoded by a topological map. Darts are connected between tiles through $\delta_{1}(\operatorname{arcs})$ and $\delta_{2}$ (segments) operators.

The root of the tree encodes the background of the image and is called the infinite region (denoted by $r_{\infty}$ ).

\section{$2.2 \quad$ Tiled topological maps}

A tiled extension of the topological map model which decomposes a map into smaller elements called tiles has been proposed to overtake memory issues induced by the representation of large partitions [4]. Indeed, tiles may be swapped on disk when they are not being processed. A topological tile is a geometric subdivision of a topological map whose interpixel matrix only encodes a subdivision of the image. Adjacency relationships between topological tiles are encoded by equivalent interpixel cells on tiles' shared borders: two pointels are equivalent if they have the same coordinates in the image referential and two linels are equivalent if their incident pointels are equivalent (Figure 2(b)). Darts' embeddings are encoded within the interpixel framework as sequences of pointels and linels. Consequently, two darts are said to have an equivalent embedding if their embeddings correspond to equivalent sequences of pointels and linels.

Let $\mathcal{D}^{t}$ be the set of darts that belong to a tile $t$ and $\mathcal{D}_{\partial}^{t, t^{\prime}}$ be the subset of $\mathcal{D}^{t}$ composed of darts that are incident to the border of $t$ and whose embedding has equivalent cells in $t^{\prime}$. Connections between darts of two adjacent tiles $t$ and $t^{\prime}$ are represented through a bijection $\varphi^{t, t^{\prime}}: \mathcal{D}_{\partial}^{t, t^{\prime}} \rightarrow \mathcal{D}_{\partial}^{t^{\prime}, t}$ that maps each dart $d$ of $\mathcal{D}_{\partial}^{t, t^{\prime}}$ to a dart $d^{\prime}$ of $\mathcal{D}_{\partial}^{t^{\prime}, t}$ such that $d$ and $d^{\prime}$ have equivalent embeddings (Figure 2(c)). Note that such a bijection can always be obtained if we consider a decomposition of the tiles' borders into basic darts whose embedding is a single couple (pointel, linel).

Besides, the decomposition in tiles entails that some darts of a tile's border may encode the tile's decomposition of the image without encoding a real border between regions. In this case, the border is considered as a fictive border. Otherwise, the 

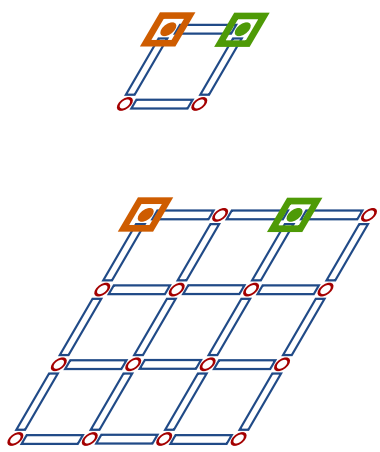

(a) $Q=1 \times 1 / 4$
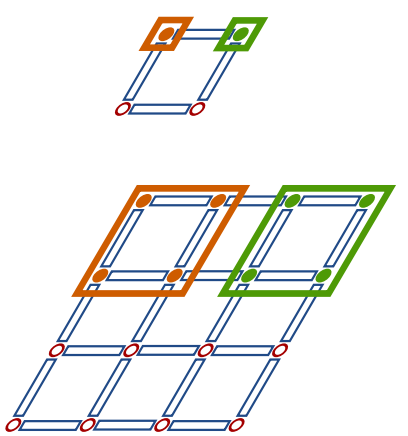

(b) $Q=2 \times 2 / 4$
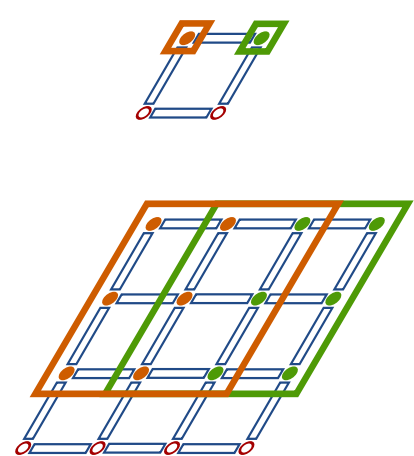

(c) $Q=3 \times 3 / 4$

Figure 3: Reduction windows of pointels within the interpixel representation of a pyramid of images. (a) Non-overlapping holed pyramid. (b) Non-overlapping pyramid without holes. (c) Overlapping pyramid.

border is called a real border. Thus, two additional operators $\delta_{1}$ and $\delta_{2}$ are introduced in order to skip darts encoding fictive borders which leads to the definition of the tiled map model (Definition 2).

Definition 2 (Tiled topological map). Let $T$ be a set of topological tiles. Let $\mathcal{D}$ be the subset of $\mathcal{D}^{T}$ whose darts belong to a real border. A tiled topological map (or tiled map) is a triplet $M=\left(\mathcal{D}, \delta_{1}, \delta_{2}\right)$ where:

- $\delta_{2}$ is an involution on $\mathcal{D}$ such as: $\delta_{2}(d)= \begin{cases}\varphi^{t, t^{\prime}}(d) & \text { if } d \in \mathcal{D}_{\partial}^{t, t^{\prime}} \\ \beta_{2}(d) & \text { otherwise }\end{cases}$

- $\delta_{1}$ is a permutation on $\mathcal{D}$ such as: $\delta_{1}(d)=\beta_{1} \circ\left(\delta_{2} \circ \beta_{1}\right)^{k}(d)$ with $k=\min \left\{p \in \mathbb{N} \mid \beta_{1} \circ\left(\delta_{2} \circ \beta_{1}\right)^{p}(d) \in \mathcal{D}\right\}$

Since $\delta_{1}$ and $\delta_{2}$ operators respectively define a permutation and an involution on $\mathcal{D}$ [5], the triplet $M=\left(\mathcal{D}, \delta_{1}, \delta_{2}\right)$ is a combinatorial map.

\subsection{Tiled top-down pyramids}

Tiled maps allow to represent partitions from large images. However, such images usually contain different information at different resolutions hence requiring the design of a multi-resolution hierarchical data structure, such as the tiled top-down pyramidal model [5].

In this paper, we propose a novel approach for the definition of tiled top-down pyramids based on the combination of three hierarchical data structures: a pyramid of images encoding a multi-resolution image, a pyramid of tiles with a constant size and a pyramid of tiled maps.

A regular pyramid of images [1] is a sequence $\left(I^{0}, \ldots, I^{n}\right)$ of images with exponentially increasing resolutions such that the reduction factor $r$ encoding the ratio 
between the size of two successive images remains constant along the pyramid. Each pixel in an image $I^{k}$ is related to a connected set of pixels in $I^{k+1}$ called its reduction window and encoded as a $M \times N$ window. The value of a pixel in $I^{k}$ is deduced from the values of pixels within its reduction window using a reduction function. Different types of pyramids may be distinguished according to the ratio $Q=M \times N / r$ : holed pyramids $(Q<1)$, non overlapping pyramids without holes $(Q=1)$ and overlapping pyramids $(Q>1)$.

Such a pyramid may be implicitly associated to a stack of matrices of inter pixel elements, also defining a regular pyramid with a same reduction factor. The notion of reduction window on such a pyramid may be adapted as follows: the reduction window of a pointel $p$ (or $\mathcal{R} \mathcal{W}(p))$ at level $k(0 \leq k<l)$ is an $M^{\prime} \times N^{\prime}$ window defined at level $k+1$ and corresponding to the set of pointels encoded by $p$ at level $k$. A hierarchical relationship is thus induced between pointels, each pointel being the father of the pointels within its reduction window. Different types of pyramids of pointels may be distinguished according to the ratio $M^{\prime} \times N^{\prime} / r$ (Fig. 3). In the following, we use non overlapping pyramids without holes both for images and pointels pyramids.

Let us now consider a given tile's size and perform a tiling of each image of a non overlapping image pyramid without holes $\mathcal{I}=\left(I^{0}, \ldots, I^{n}\right)$. Each tile defined in an image $I^{k}$ is expanded into $r \times r$ tiles in $I^{k+1}$, where $r$ denote the reduction factor of $\mathcal{I}$. Hierarchical relationships established within an image pyramid induce thus hierarchical relationships between tiles decomposing each image of the pyramid. This last hierarchy is called a pyramid of tiles (Definition 3).

Definition 3 (Pyramid of tiles). Let I be an image. A pyramid of tiles associated with $I$ is a couple $\left(\mathcal{I}, \mathcal{T}=\left(T^{0}, \ldots, T^{n}\right)\right)$ where $\forall k, 0 \leq k<n$ :

- $\mathcal{I}=\left(I^{0}, \ldots, I^{n}\right)$ is a non-overlapping pyramid of images without holes associated with $I$;

- $T^{k}=\{t(i, j, k)\}_{(i, j) \in\left[0, l^{k} / l\left[\times\left[0, h^{k} / h[\right.\right.\right.}$ is a rectangular tiling of the $l^{k} \times h^{k}$ image $I^{k}$ by a set of $l \times h$ tiles $t(i, j, k)$;

- $\mathcal{R} \mathcal{W}(t(i, j, k))=\left\{t\left(i^{\prime}, j^{\prime}, k+1\right) \mid\left\lfloor i^{\prime} / r\right\rfloor=i,\left\lfloor j^{\prime} / r\right\rfloor=j\right\}$ is the reduction window of tile $t(i, j, k)$, where $\lfloor$.$\rfloor denotes the floor operator.$

While both pyramids of images and tiles are regular hierarchical data structures, our aim is to define an irregular pyramid of tiled maps. A tiled top-down pyramid (Definition 4) is thus a stack of finer and finer partitions, defined at different resolutions and encoded by tiled topological maps. The hierarchy is represented by up/down relationships between darts and regions [4]. Moreover, since each map $G^{k+1}$ of the pyramid is finer than $G^{k}$, a border defined at a given level of the pyramid cannot be removed at levels below. A tiled top-down pyramid is thus a causal structure [6]. Consequently, we propose a new definition for tiled top-down pyramids (Definition 4) where the tiled maps (levels) are based on the tiling of a given pyramid of tiles. 


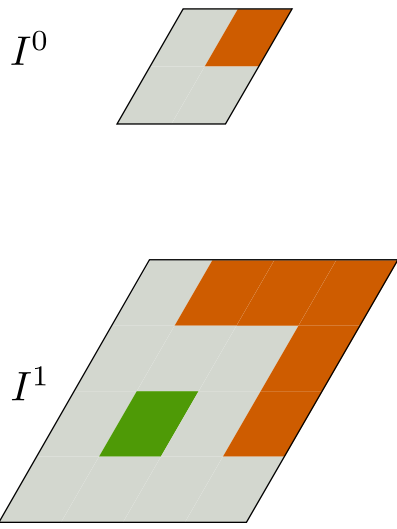

(a)

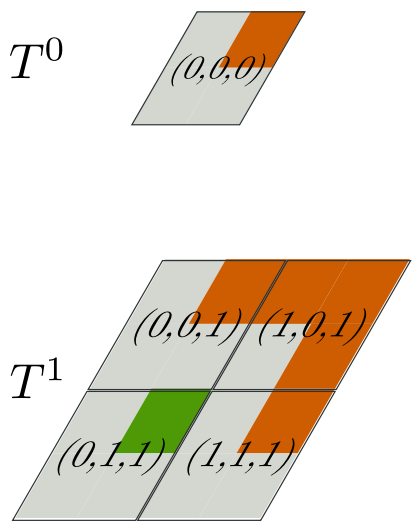

(b)

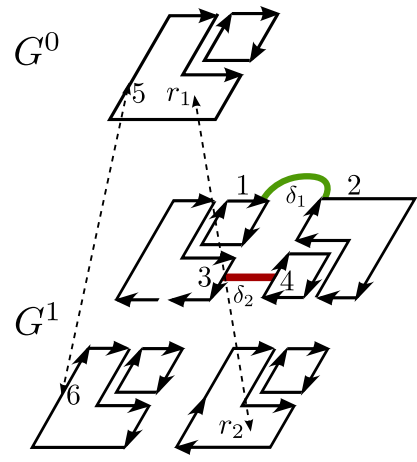

(c)

Figure 4: Tiled top-down pyramids: a combination of three hierarchical models for the representation of large multi-resolution images. (a) Pyramid of images $(r=2)$. (b) Pyramid of tiles. (c) Pyramid of tiled maps. Dotted arrows illustrate up/down relationships between darts and regions. Darts that belong to $r_{\infty}$ are not represented.

Definition 4 (Tiled top-down pyramid). Let I be an image. A tiled top-down pyramid is a triplet $\left(\mathcal{I}, \mathcal{T}, \mathcal{G}=\left(G^{0}, \ldots, G^{n}\right)\right)$ where $\forall k, 0 \leq k<n$ :

- $(\mathcal{I}, \mathcal{T})$ is a pyramid of tiles composed of $n+1$ levels and associated with $I$;

- $G^{k}$ is a tiled map corresponding to the tiling of $T^{k}$;

- $G^{k+1}$ is defined on $\left(I^{k+1}, T^{k+1}\right)$ and is deduced from $G^{k}$ by decomposition operations (e.g. splitting of faces of $G^{k}$ ) in order to be finer than $G^{k}$.

The construction scheme of a tiled top-down pyramid may rely on the notion of focus of attention which allows to refine only regions of interest identified in the upper levels of the pyramid. The advantage of such a construction scheme is twofold since it may reduce memory usage and processing time while imitating experts' analysis scheme. Finally, note that within a given application field, only some specified levels of the pyramid may need to be explicitly encoded.

\section{Segmentation scheme}

We propose a generic segmentation scheme for large multi-resolution images based on the tiled top-down pyramid framework. Our segmentation process relies on the definition of an Oracle that may combine several criteria of different natures to take advantage of geometrical, topological and hierarchical features. Since this segmentation step is part of the global construction scheme of the tiled top-down pyramid, it must neither impact the causality of the pyramid nor its refinement by focus of attention. The process assumes an initial partition $G^{0}$ which may be a single region or which may result from an extraction algorithm $[2,3]$ at resolution $I^{0}$. In both 
cases, a partition at level $k+1$ is deduced from level $k$ by applying the following two steps procedure:

- a projection step of the regions' borders of $G^{k}$ onto $G^{k+1}$ which preserves the topology of $G^{k}$ and expands the geometry of $G^{k+1}$ borders according to the reduction factor.

- an Oracle-based refinement step, restricted to regions whose father is a region of interest in the previous level (focus of attention).

Figure 5 illustrates the main steps of our projection procedure. The main issue is to project the former borders onto the current resolution while preserving the same topology. Intuitively, the projection of a border $b=\left(p_{1}, \ldots, p_{m}\right)$ in $G^{k}$ is a border $b^{\prime}$ in $G^{k+1}$ contained within the strip formed by the reduction windows of the pointels $\left(p_{i}\right)_{i=1, m}$ of $b$. This strip is a connected set since the pyramid of images contains no holes. Moreover, the pyramid is not overlapping and therefore, two projected borders cannot intersect, except on their extremities which correspond to the intersection of several borders. Let us consider a pointel $p_{1}$ in $G^{k}$ which corresponds to an intersection of borders and its projection $P\left(p_{1}\right)$ in $G^{k+1}$. In order to keep the same topology in $G^{k}$ and $G^{k+1}$, we define the projection of each linel in $G^{k}$ incident to $p_{1}$ as an horizontal or vertical straight line of length $r$ in $G^{k+1}$, incident to $P\left(p_{1}\right)$, and with the same direction than the initial linel. These straight lines correspond to a standard linel's projection which guarantees that $P\left(p_{1}\right)$ is the only intersection point of projected borders within $p_{1}$ 's reduction window. This standard projection is also performed on $p_{m}$ and the connection between the two straight lines incident to $P\left(p_{1}\right)$ and $P\left(p_{2}\right)$ is achieved using a Dijkstra algorithm within the strip formed by the reduction windows of pointels $\left(p_{i}\right)_{i=2, m-1}$. Dijkstra algorithm is applied with an edge weight proportional to the inverse of the gradient computed in $I^{k+1}$, hence taking into account the additional information provided by $I^{k+1}$.

The refinement step relies on the definition of the Oracle proposed in Algorithm 1: according to a segmentation criterion (line 3), our scheme refines a region if its father in the previous level is a region of interest (line 2) while preserving the causality of the model (line 1).

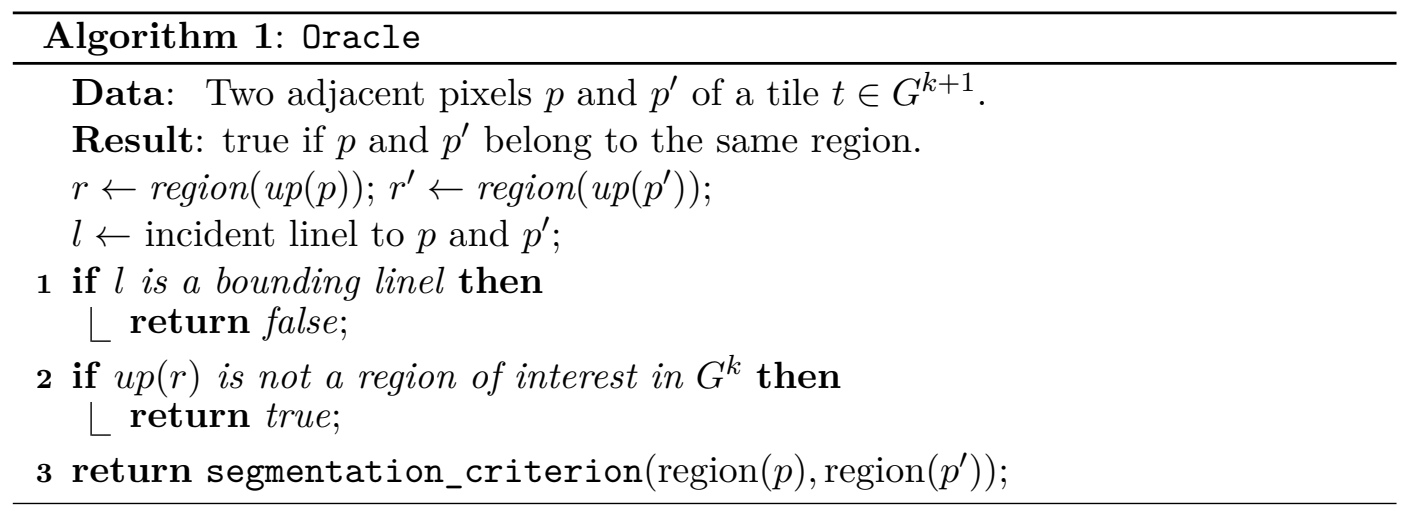



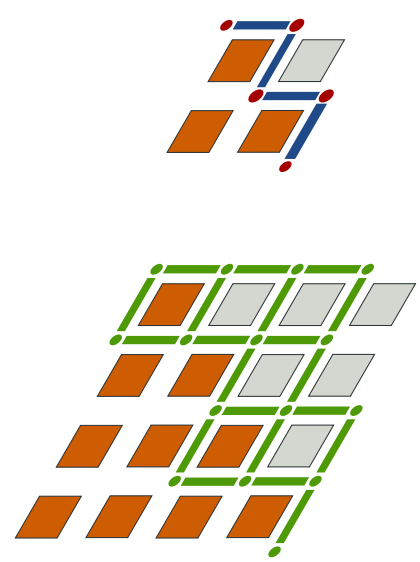

(a)
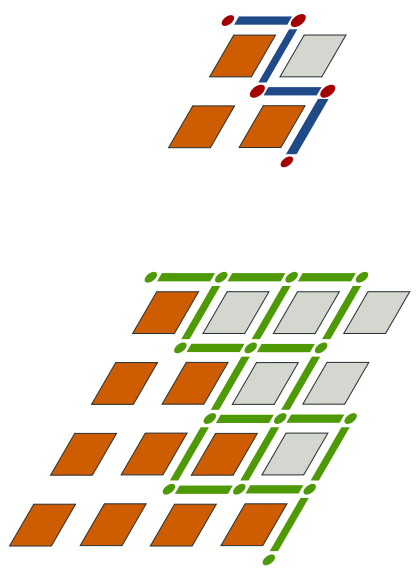

(b)
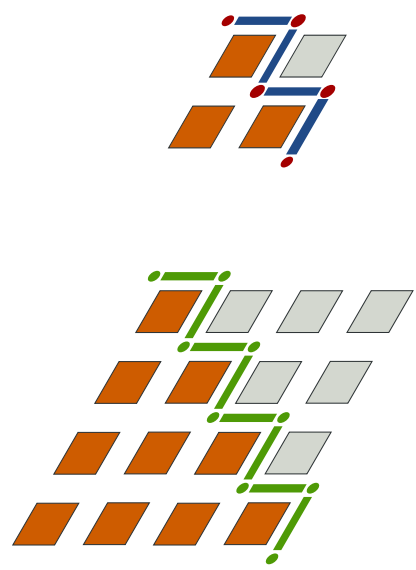

(c)

Figure 5: Projection of regions borders. (a) Strip composed by the reduction windows of the pointels that belong to the border of the previous level. (b) Restrictions on borders extremities. (c) Shortest path according to a given energy.

\section{Application for large histological images segmentation}

We propose a practical use case ${ }^{4}$ of the top-down framework for the segmentation of large histological images. Our objective is to demonstrate the generic aspect of our model with the integration of an existing segmentation scheme. In this application, histological images are produced as a stack of three images with increasing resolutions. Their manual analysis by pathologists are performed using a focus of attention over regions of interest up to the full resolution image where cells' mitosis are enumerated. This estimation is then used for breast cancer diagnosis grading.

Our top-down segmentation algorithm uses the same scheme than [9] with the additional use of a tiled top-down pyramid (Figure 6). Successive $k$-means based classifications [7] allow to label and refine regions of interest. At resolution 1, the background is separated from tissue. Then, a classification in two classes of tissue at resolution 2 allows to extract lesions and a last classification distinguishes the cancer in situ from stroma at resolution 3 .

Table 1 presents experimental results for the segmentation of a representative histological image. Column 2,3 and 4 respectively indicate the size of the images, the number of tiles and the number of regions of their associated partitions. In Column 5 and 6 , we provide runtimes for both pyramid construction scheme (model) and our segmentation algorithm (segmentation). These results demonstrate that our model does not introduce an important additional cost while providing an efficient access to hierarchical, topological and geometrical features. Note that runtimes for

\footnotetext{
${ }^{4}$ Our model is implemented in $\mathrm{C}++$ and computations are carried out on an Intel E5300@2GHz with 2GB RAM.
} 


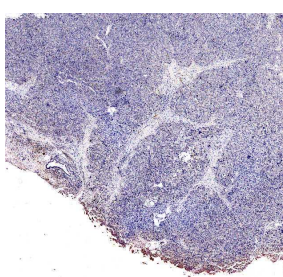

(a)

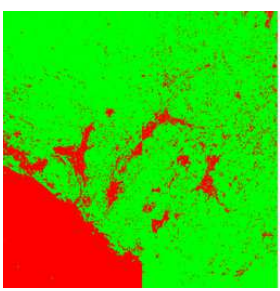

(b)

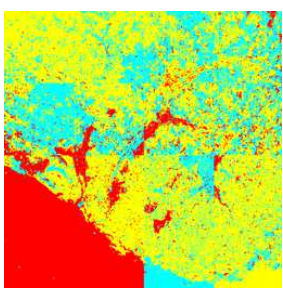

(c)

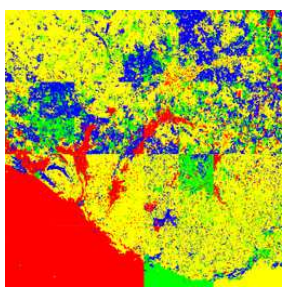

(d)

Figure 6: Classification scheme of histological images. (a) Original image. (b) Resolution 1: distinction between tissue and background. (c) Resolution 2: distinction between lesions and tissue. (d) Resolution 3: distinction between cancer in situ and stroma.

Table 1: Segmentation of an histological image within our top-down framework.

\begin{tabular}{|c|c|c|c|c|c|c|}
\hline \multirow[t]{2}{*}{ level } & \multirow{2}{*}{$\begin{array}{c}\text { size } \\
\text { (pixels) }\end{array}$} & \multirow{2}{*}{$\begin{array}{l}\text { number } \\
\text { of tiles }\end{array}$} & \multirow{2}{*}{$\begin{array}{l}\text { number } \\
\text { of regions }\end{array}$} & \multicolumn{2}{|c|}{ runtime (s) } & \multirow{2}{*}{$\begin{array}{l}\text { ram } \\
(\mathrm{MB})\end{array}$} \\
\hline & & & & model & segmentation & \\
\hline 1 & 250 & 16 & 574 & 9.8 & 35.2 & 104 \\
\hline 2 & 5 & 64 & 13 & 43.6 & 186.6 & 101 \\
\hline 3 & $10000 \times 10000$ & 256 & 225672 & 267.8 & 645.0 & 106 \\
\hline
\end{tabular}

levels' extraction are linear with the size of the image but slightly increase with the number of tiles due to disc access delays. Finally, tiled maps allow to preserve a constant memory usage around 100MB (Column 7) with a tile size of $625 \times 625$ pixels.

\section{Conclusion}

In this paper, we have proposed a new approach to the definition of tiled top-down pyramids which emphasizes the combination of regular and irregular hierarchical data structures. We have described a segmentation scheme with a new projection step which takes into account the additional information provided by each image of the pyramid. Finally, we have demonstrated the efficiency of our framework with an application on histological images.

This work opens interesting perspectives such as the definition of topological criteria that could be combined to the present segmentation process. Those criteria may enhance results by taking further advantage of our top-down framework. Finally, a medical evaluation should be performed to confirm the accuracy of the partitions provided by our method. 


\section{Acknowledgements}

This research is part of the FoGrImMi project, supported by the ANR foundation under grant ANR-06-MDCA-008-01/FOGRIMMI.

\section{References}

[1] M. Bister, J. Cornelis, and A. Rosenfeld. A critical view of pyramid segmentation algorithms. Pattern Recognition Letters, 11(9):605-617, 1990.

[2] L. Brun, M. Mokhtari, and J.P. Domenger. Incremental modifications on segmented image defined by discrete maps. Journal of Visual Communication and Image Representation, 14:251-290, 2003.

[3] G. Damiand, Y. Bertrand, and C. Fiorio. Topological model for two-dimensional image representation: definition and optimal extraction algorithm. Computer Vision and Image Understanding, 93(2):111-154, 2004.

[4] R. Goffe, L. Brun, and G. Damiand. Tiled top-down combinatorial pyramids for large images representation. International Journal of Imaging Systems and Technology, 21(1):28-36, 2011.

[5] R. Goffe, G. Damiand, and L. Brun. A causal extraction scheme in topdown pyramids for large images segmentation. In 13th International Workshop On Structural and Syntactic Pattern Recognition, number 6218, pages 264-274. LNCS, 2010.

[6] L. Guigues, J.P. Cocquerez, and H. Le Men. Scale-sets image analysis. International Journal of Computer Vision, 68(3):289-317, 2006.

[7] T. Kanungo, D.M. Mount, N.S. Netanyahu, C.D. Piatko, R. Silverman, and A.Y. Wu. An efficient k-means clustering algorithm: Analysis and implementation. IEEE Trans. on PAMI, 24(7):881-892, 2002.

[8] V.A. Kovalevsky. Finite topology as applied to image analysis. Computer Vision, Graphics, and Image Processing, 46(2):141-161, 1989.

[9] V. Roullier, O. Lézoray, V.T. Ta, and A. Elmoataz. Mitosis extraction in breastcancer histopathological whole slide images. In $I S V C, 2010$. 